\title{
Near-Term Decrease in Brain Volume following Mild Traumatic Injury Is Detectible in the Context of Preinjury Volumetric Stability: Neurobiologic Insights from Analysis of Historical Imaging Examinations
}

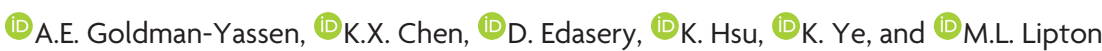

\begin{abstract}
BACKGROUND AND PURPOSE: Neurodegeneration after mild traumatic brain injury may manifest as decreasing regional brain volume that evolves from months to years following mild traumatic brain injury and is associated with worse clinical outcomes. We hypothesized that quantitative brain volume derived from CT of the head, performed for clinical indications during routine care, would change with time and provide insights into the putative neuroinflammatory response to mild traumatic brain injury.
\end{abstract}

MATERIALS AND METHODS: We searched the electronic medical record of our institution for NCCTs of the head performed in patients with mild traumatic brain injury and included those who also underwent NCCTs of the head 1 month to 1 year before and after mild traumatic brain injury for an indication unrelated to trauma. Controls underwent 3 sequential NCCTs of the head with indications unrelated to trauma. The whole-brain and intracranial volume groups were computed using ITK-SNAP. Brain volumes normalized to intracranial volumes were compared across time points using the Wilcoxon signed-rank test.

RESULTS: We identified 48 patients from 2005 to 2015 who underwent NCCTs of the head in the emergency department for mild traumatic brain injury and had NCCTs of the head performed both before and after mild traumatic brain injury. Median normalized brain volumes significantly decreased on the follow-up study post-mild traumatic brain injury (0.86 versus $0.84, P<.001)$ and were similar compared with pre-mild traumatic brain injury studies ( 0.87 versus $0.86, P=.927)$. There was no significant difference between normalized brain volumes in the 48 controls.

CONCLUSIONS: A decrease in brain volume following mild traumatic brain injury is detectable on CT and is not seen in similar patients with non-mild traumatic brain injury during a similar timeframe. Given the stability of brain volume before mild traumatic brain injury, CT volume loss may represent the subtle effects of neurodegeneration.

ABBREVIATIONS: IQR = interquartile range; $\mathrm{mTBI}=$ mild traumatic brain injury; $\mathrm{NCCTH}=\mathrm{NCCT}$ of the head; $\mathrm{TBI}=$ traumatic brain injury

$\mathbf{T}$ raumatic brain injury (TBI) affects an estimated 1.7 million patients annually in the United States and is a major cause of morbidity and mortality. ${ }^{1,2}$ Most, $70 \%-90 \%$ of cases of TBI, are classified as mild TBI (mTBI), which is also known as concussion. ${ }^{3}$ Although mTBI-related symptoms resolve in most

Received March 27, 2018; accepted after revision June 29.

From the Department of Radiology (A.E.G.-Y., K.X.C., D.E., K.H.), Montefiore Medical Center, Bronx, New York; Department of Epidemiology and Population Health (K.Y.), Albert Einstein College of Medicine, Bronx, New York; and Gruss Magnetic Resonance Research Center Departments of Radiology, Psychiatry and Behavioral Sciences and Dominick P. Purpura Department of Neuroscience (M.L.L.), Albert Einstein College of Medicine, Bronx, New York.

Preliminary results were presented at: Annual Meeting of the American Society of Neuroradiology and the Foundation of the ASNR Symposium, April 22-27, 2017; Long Beach, California.

Please address correspondence to Michael L. Lipton, MD, PhD, Albert Einstein College of Medicine, 1300 Morris Park Ave, MRRC, Room 219C, Bronx, NY 10461; e-mail: michael.lipton@einstein.yu.edu; @AdGoldmanYassen

http://dx.doi.org/10.3174/ajnr.A5769 patients, a minority of patients experience persistent symptoms, which may be disabling. ${ }^{4}$

Conventional CT and MR imaging examinations performed as part of routine clinical care typically reveal no visible indications of trauma in patients with mTBI. Neuroinflammation, proposed as a mechanism underlying persistent dysfunction and brain structural changes following mTBI, however, might manifest as subtle brain swelling on acute timeframe imaging. ${ }^{5} \mathrm{Al}$ though quantitative studies of brain volume have demonstrated both gray and white matter volume loss after mTBI using MR imaging, published studies of brain volume in TBI have an important limitation: They did not have access to pre-mTBI brain imaging and cannot therefore assess change of brain volume from a preinjury baseline. ${ }^{6-16}$ Moreover, prior studies generally reported change during the long-term after mTBI. Although prior studies have shown a reduction in brain volume after mTBI, in the absence of a baseline, it is unclear whether these changes are the 

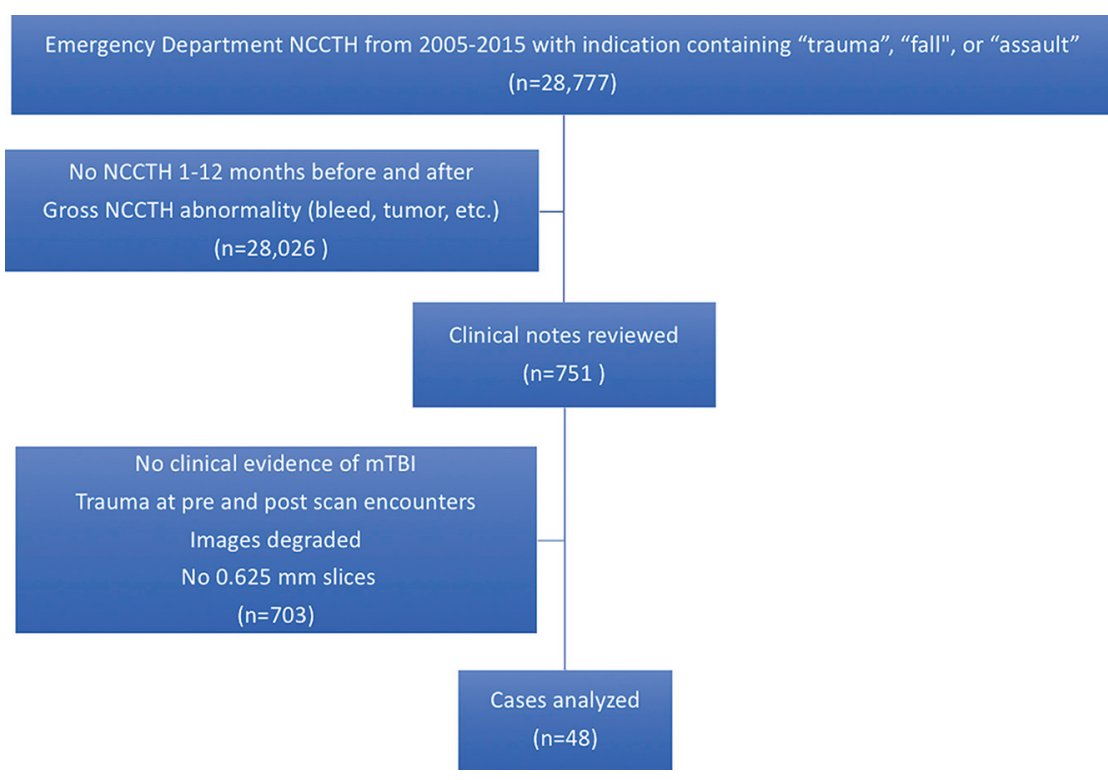

FIG 1. Flowsheet of included and excluded cases.

result of resolving subtle trauma-related edema due to neuroinflammation or atrophy from neurodegeneration.

CT is widely performed as the initial imaging test of choice for patients who present with acute mTBI, but it has not been reported as a method to quantify brain volume changes. ${ }^{17-19}$ In this study, we aimed to mine existing clinical CT examinations, performed as a part of routine clinical care, to quantify baseline brain volume before mTBI and assess subsequent brain volume change. Because NCCT of the head (NCCTH) is ordered in patients for a variety of reasons unrelated to trauma, we can compare mTBI examinations with CT performed both before and following mTBI in the same patients, to quantify brain volume changes following mTBI and determine whether the change suggests resolution of acute swelling, consistent with resolving neuroinflammation, or loss of volume, consistent with neurodegeneration.

\section{MATERIALS AND METHODS \\ Study Population and Study Design}

After obtaining approval from our institutional review board, including a waiver of informed consent for our Health Insurance Portability and Accountability Act-compliant study, we used Clinical Looking Glass (Streamline Health, Atlanta, Georgia) to search our electronic medical records for patients who underwent NCCTH for the evaluation of mTBI. Specifically, we searched for radiology reports from NCCTHs ordered in the emergency department at Montefiore Medical Center from 2005 to 2015 with clinical indications of "trauma," "fall," "concussion," or "assault." Subjects were included if they also underwent NCCTH 1-12 months before and after the mTBI scan for a nontraumatic indication (Fig 1). If the patient underwent multiple scans in these intervals, the scan closest to the time of mTBI was used. Controls were identified by searching Clinical Looking Glass for patients who underwent 3 sequential NCCTHs in the emergency department for nontraumatic reasons at time intervals similar to those in the patients with mTBI.

Demographic information and medical comorbidities were obtained for each individual from the electronic medical records at the time of mTBI (cases) or the second CT (controls). Clinical notes associated with NCCTHs performed before mTBI, at the time of mTBI, and after mTBI were reviewed to assess the clinical state of the patient at the time of each scan, including the mechanism of injury, clinical indications, and signs and symptoms.

\section{CT Image Acquisition and Data Storage}

During routine clinical care, axial NCCTH images were acquired on LightSpeed VCT and LightSpeed RT16 (GE Healthcare, Milwaukee, Wisconsin) at a kilovolt (peak) ranging from 120 to 140 and milliampere ranging from 280 to 300. For each subject, the hardware and technique remained constant across all time points. We retrieved DICOM files containing $0.625-\mathrm{mm}$-thick axial slices of the head from the PACS and stored them on a password-protected local hard drive. Subjects were excluded if 0.625-mm-thick images were not available.

\section{Image Processing}

Processing and analysis were performed by 3 postgraduate year 3 or postgraduate year 4 radiology residents, supervised by an American Board of Radiology Certificate of Added Qualification-certified neuroradiologist. Before the processing and analysis of all cases, a random subset of 18 cases was independently segmented by 2 residents and interrater reliability was assessed. Once the protocol was considered reliable, the residents then processed and analyzed the remaining cases independently to conserve time and resources. Incomplete datasets were excluded. Next, all images were first visually inspected. Subjects were excluded if there was evidence of acute or active disease, including hemorrhage, mass, acute or chronic infarct, and hydrocephalus. Images degraded by motion or beamhardening artifacts were also excluded. Patients with age-related abnormalities, such as white matter hypodensities, were not excluded.

We assessed total brain and intracranial volumes using semiautomated threshold segmentation in ITK-SNAP, Version 3.6.0 (www.itksnap.org), adhering to the following standardized protocol for consistency ${ }^{20}$ : An initial lower threshold of $20 \mathrm{HU}$ and an upper threshold of $75 \mathrm{HU}$ were applied. We chose these thresholds on the basis of maximal differentiation between brain parenchyma and CSF on visual inspection. We then placed 10-mm seeds in the centrum semiovale, corona radiata, basal ganglia, adjacent to the occipital horn of each lateral ventricle, and pons. Seeds were grown to fill the desired volume, followed by inspection of the segmentations and growing of additional seeds to fill parenchyma not included by active contour evolution. Structures spuriously included in the initial segmentation, such as paraspinal soft tissues or the optic nerve, were then manually edited. The inferior extent of the cerebellar tonsils was defined as the caudal limit of the segmentation volume. A representative final segmen- 


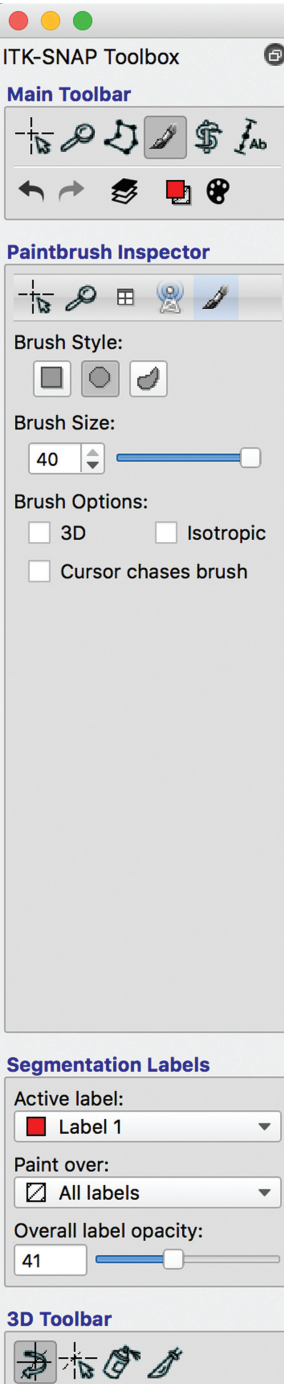

类植 8 ser004img00001.dcm - New Segmentation - ITK-SNAP
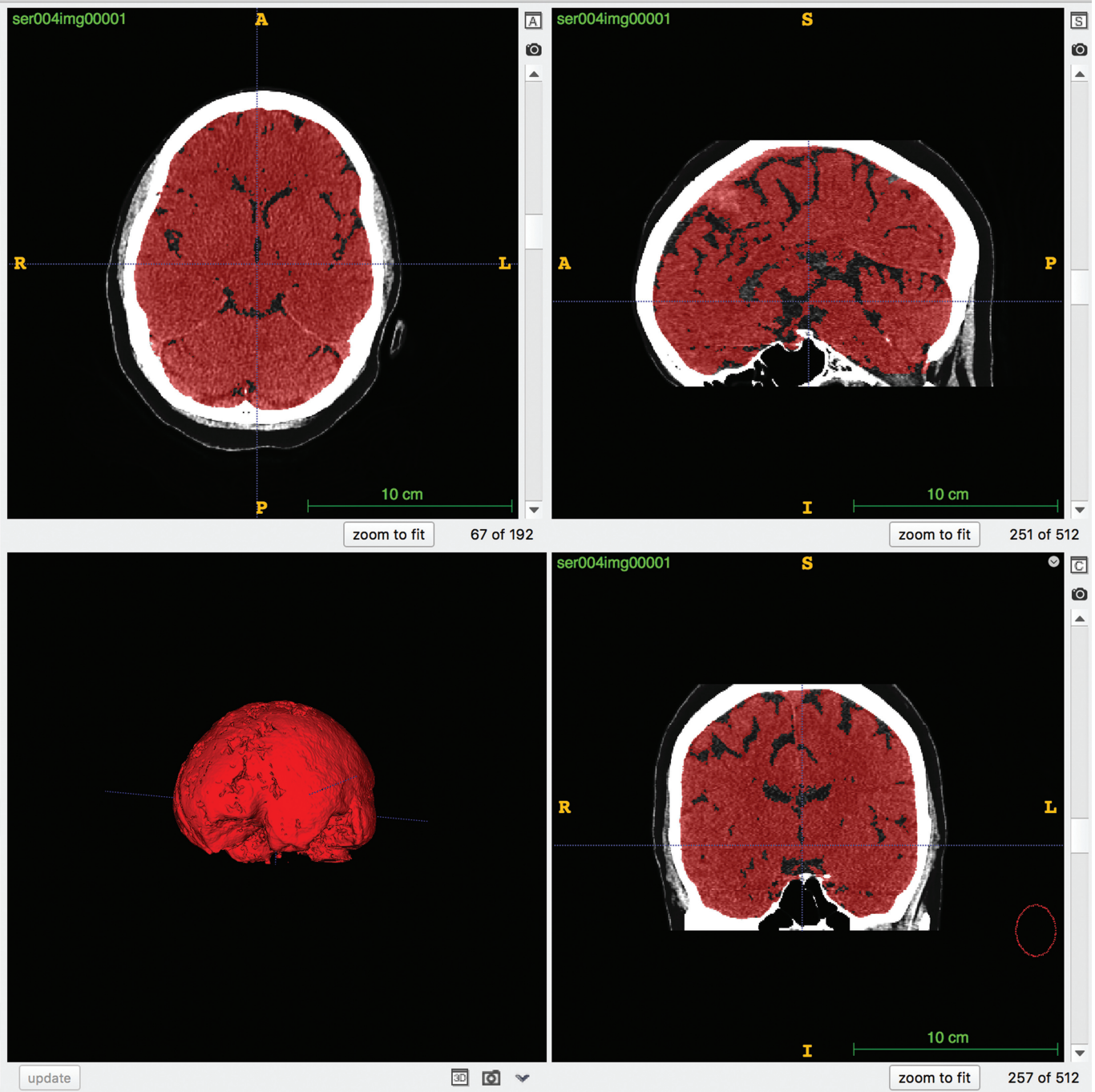

FIG 2. ITK-SNAP screenshot showing total brain volume segmentation.

tation is demonstrated in Fig 2. To calculate total intracranial volume, we started with the previously computed brain segmentation. We then used a lower threshold of $-20 \mathrm{HU}$ and an upper threshold of $100 \mathrm{HU}$ to maximize differentiation between the skull and intracranial contents. The segmentation was then grown to fill the intracranial volume, and structures spuriously included, such as paraspinal soft tissues or the optic nerve, were then manually edited. The inferior extent of the cerebellar tonsils was also defined as the caudal limit of the intracranial segmentation volume.

\section{Statistical Analysis}

Statistical analysis was performed using STATA, Version 12.1 (StataCorp, College Station, Texas). Each individual's brain volume was initially normalized to his or her total intracranial volume. Continuous demographic variables were compared using the Mann-Whitney $U$ test, and categoric variables, with the $\chi^{2}$ or Fisher's exact test when appropriate. Interrater reliability was assessed using the intraclass correlation coefficient. Brain volumes were compared within individuals across timepoints using the Wilcoxon signed rank test. Absolute changes in brain volumes were calculated between the initial scan and the index scan and the index scan and follow-up for both the patients and controls. Differences in absolute volume changes between patients and controls were calculated using the Mann-Whitney $U$ test. A 2-tailed $P$ value $<.05$ was considered statistically significant.

\section{RESULTS}

We identified 28,777 patients who underwent NCCTH ("index CT") in 1 emergency department for evaluation of traumatic head injury. Of these, 751 also underwent NCCTH within 1-12 months both before ("baseline CT") and after ("follow-up CT") the index CT (Fig 1). Of the 751 patients, 48 patients had no visible CT abnormalities on any CT or history of head trauma at the time of either the baseline CT or follow-up CT. In addition, 48 matched control subjects were identified who underwent 3 CT examinations, which were similarly named (baseline, index, and follow-up CT).

Patient demographics did not differ between patients with mTBI and controls (Table 1). Similarly, there was no significant difference in the median time between baseline, index, and fol- 


\begin{tabular}{lccc}
\hline & mTBI $(\boldsymbol{n}=48)$ & Controls $(\boldsymbol{n}=48)$ & $\boldsymbol{P}$ Value \\
\hline Median age (IQR) (yr) & $63(52-79)$ & $71(50-82)$ & $.202^{\mathrm{a}}$ \\
Male sex percent (No.) & $42 \%(20)$ & $31 \%(15)$ & $.289^{\mathrm{b}}$ \\
Median pre- to index interval (IQR) (day) & $118(67-239)$ & $171(87-274)$ & $.123^{\mathrm{a}}$ \\
Median index to post interval (IQR) (day) & $149(79-204)$ & $124(61-219)$ & $.901^{\mathrm{a}}$ \\
\hline
\end{tabular}

a Mann-Whitney $U$ test.

${ }^{\mathrm{b}} \chi^{2}$ test.

Table 2: No significant differences in comorbidities that may affect brain volume between cases and controls

\begin{tabular}{lccc} 
& $\begin{array}{c}\text { mTBI } \\
(\boldsymbol{n}=\mathbf{4 8})\end{array}$ & $\begin{array}{c}\text { Controls } \\
(\boldsymbol{n}=\mathbf{4 8})\end{array}$ & $\begin{array}{c}\boldsymbol{P} \\
\text { Value }^{\mathbf{a}}\end{array}$ \\
\hline Dementia & $23 \%(11)$ & $25 \%(12)$ & $>.999$ \\
End-stage renal disease & $8 \%(4)$ & $8 \%(4)$ & $>.999$ \\
Diabetes mellitus & $48 \%(23)$ & $38 \%(18)$ & .409 \\
Hypertension & $75 \%(36)$ & $77 \%(37)$ & $>.999$ \\
Cardiac arrhythmia/valvular & $19 \%(9)$ & $19 \%(9)$ & $>.999$ \\
$\quad$ disease & $10 \%(5)$ & $10 \%(5)$ & $>.999$ \\
Seizure disorder & $8 \%(4)$ & $6 \%(3)$ & $>.999$ \\
Cirrhosis & $30 \%(14)$ & $19 \%(9)$ & .339 \\
Psychiatric disorders & & &
\end{tabular}

low-up scans. Additionally, patients with mTBI and controls had similar rates of underlying medical comorbidities (Table 2). Among the 48 patients with mTBI, 36 fell (75\%), 5 were assaulted $(10 \%), 2$ were involved in a motor vehicle collision $(4 \%)$, and 5 sustained trauma by other mechanisms (10\%). Broadly, nontraumatic clinical indications in both mTBI and control groups included altered mental status from a variety of reasons, headache, seizure, dizziness, and weakness.

Among 18 randomly selected subjects, we found excellent agreement between 2 researchers, with intraclass correlation coefficients of 0.998 (95\% confidence interval, 0.995-0.999) for the index CT and 0.965 (95\% CI, 0.905-.986) for the follow-up CT.

When we compared subjects across time in a pair-wise fashion, brain volume (as a normalized fraction of total intracranial volume) decreased from 0.86 at the time of mTBI to 0.84 at follow-up $(P=.02$, Fig 3$)$. We found no significant change in brain volume from the baseline NCCTH to the time of mTBI and no significant change in brain volume of the controls during the same time intervals.

We next compared the trajectory of volume changes between patients with mTBI and controls. The magnitude of change in adjusted brain volume between study cases at the time of mTBI and follow-up NCCTH was significantly larger than that of controls during a similar time interval (0.012; interquartile range [IQR], $-0.001-0.027$ versus 0.002 ; IQR, $-0.008-0.016 ; P=$ $.021)$. There was no difference in the magnitude of adjusted brain volume change between the baseline NCCTH for patients with $\mathrm{mTBI}$ as well as controls during the same time interval $(0.000$; IQR, $-0.007-0.010$ versus 0.004 ; IQR, $-0.014-0.013 ; P=.753)$. Brain volume declined from the baseline CT following mTBI significantly more than the change for controls during the same time interval (0.007; IQR, $-0.001-0.026$ versus -0.002 ; IQR, - 0.015$0.020 ; P=.034)$.

\section{DISCUSSION}

Although CT is the recommended initial method for evaluating patients with mild traumatic brain injury, to our knowledge, this is the first report of quantitative analysis of brain volume changes derived from NCCTH in patients with mTBI. ${ }^{21}$ While we do not propose the use of CT for routine clinical follow-up, the ubiquity of NCCTH performed during routine clinical practice in the emergency department makes these data ripe for analysis to characterize and gain insight into the underlying disease process. We found a significant decrease in brain volumes from the time of mTBI to follow-up 1-12 months later, with evidence of stable brain volume during 1-12 months before mTBI and across similar timeframes in controls evaluated in the emergency department for reasons other than TBI.

Changes in brain volume following mTBI have been described in many previous studies to correlate with clinical sequelae of long-term brain injury. In 2 studies that reported whole-brain volume changes in patients with TBI with a range of severity, average total brain volume decreases of $7.6 \mathrm{~mL}$ were found at 1 year in patients with $\mathrm{mTBI}$ and $157.3 \mathrm{~mL}$ in patients with mild and moderate TBI. ${ }^{6,16}$ For reference, normal human total brain volume at 32 years of age averages $1273.6 \mathrm{~mL}$ in men and $1131.1 \mathrm{~mL}$ in women and declines by about $0.2 \%-0.5 \%$ per year in healthy individuals. ${ }^{22-24}$ We showed an absolute decrease of approximately $40 \mathrm{~mL}$ after mTBI at a median of 149 days' follow-up. Although our study and others showed significant brain volume changes, some studies failed to show any volume differences after mTBI. ${ }^{25,26}$ Most studies that reported changes in regional brain volume showed changes in regions such as the frontal gyri, precuneus, temporal gyri, caudate, cingulum, and hippocampus. ${ }^{6,8,15,27,28}$ The ITK-SNAP protocol we used is robust to discrimination of bone, fluid, and soft tissue on CT but cannot discriminate soft-tissue differences (eg, gray versus white matter) sufficiently to allow regional segmentation. Currently available regional segmentation algorithms such as FreeSurfer (http://surfer.nmr.mgh.harvard.edu) are validated for parcellation of MR imaging. ${ }^{29} \mathrm{CT}$ images provide substantially lower soft-tissue contrast than MR imaging, and we are not aware of any validated methods for parcellation of brain regions based on CT.

Although decreases in brain volume have been reported after mTBI, it is unclear if these changes reflect resolution of traumarelated edema, which might be at a level not detectable on visual inspection, or development of atrophy in the wake of injury. Animal models have shown both early cortical thickening due to transient edema after trauma followed by reduction of cortical thickness due to atrophy. ${ }^{30,31}$ Our findings of stable brain volume between pretrauma and index mTBI scans with subsequent decreases in volume at follow-up suggest neurodegeneration following mTBI rather than acute edema at the time of mTBI with subsequent resolution. Most volumetric studies of mTBI do not include comparison with preinjury imaging. However, a longitudinal study of ice hockey players included MR imaging at the beginning and end of a season of play as well as additional scans for players who sustained mTBI during that time. Brain volumes at the end of the hockey season declined in all players, regardless of incident $\mathrm{mTBI}$, and no increase in brain volume 


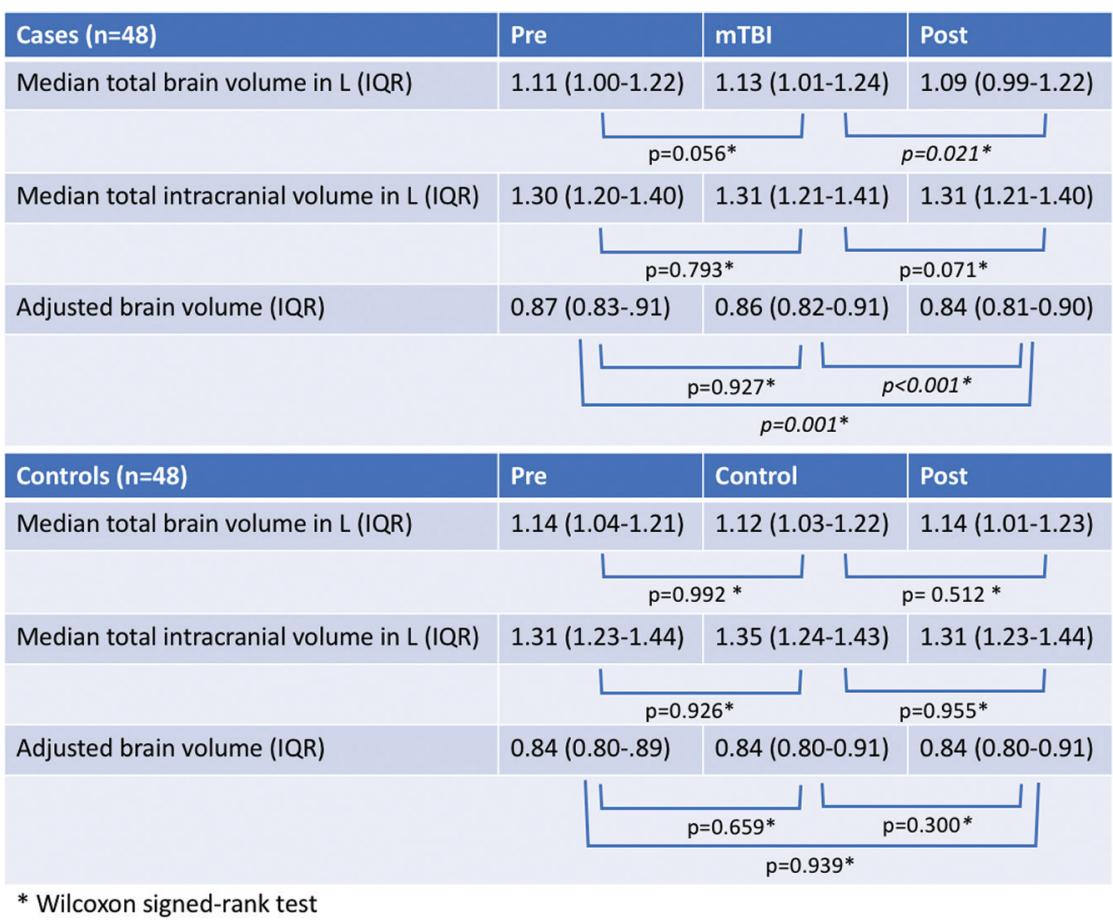

FIG 3. Differences in brain volume across time points in cases and controls. in volume due to aging during 12 months makes it highly implausible that we would detect a change due to aging in our follow-up interval (mTBI median, 267 days; control median, 295 days). Moreover, to detect a decline due to normal aging during only 1 of the 2 sequential follow-up intervals we examined would be very unlikely. Last, our CT protocols could not be optimized prospectively, creating the potential for bias due to image quality. We were, however, able to confirm that there were no changes in imaging equipment during the timeframe of each subject's 3 CT examinations, that voxel size was identical across all CT examinations, and that imaging parameters for the studies we included differed minimally, if at all. Moreover, minimal changes in imaging parameters limitation is ubiquitous in long-term studies, especially retrospective analyses. was detected at the time of mTBI. ${ }^{12}$ The decline in brain volumes regardless of concussion status in these hockey players could represent a consequence of repeat game-related brain trauma in the entire cohort.

In addition to mTBI, many other clinical disorders have been shown to cause longitudinal change of brain volume, including dehydration, schizophrenia, neurodegenerative disorders, and normal aging. ${ }^{32-36}$ Although our patient group with mTBI did exhibit comorbid medical conditions, the control group, who were of similar age, exhibited a similar prevalence of comorbid disorders but did not demonstrate any significant change in brain volume during similar time periods. This finding makes comorbid disease an unlikely explanation for the effects we found. We considered that administration of IV fluid in the emergency department might result in an increase in brain volume, with a subsequent decrease detectable on follow-up. However, because all 3 CT examinations were performed in the emergency department but the only significant change occurred between the index and follow-up CT, and only in patients with mTBI, we consider hydration status an unlikely explanation of the findings.

Our study has several limitations. First, the retrospective design cannot confirm a causal relationship between incident mTBI and brain volume change. Second, our reliance on clinical information collected as a part of routine clinical care may be inadequate to determine the true nature of a patient's clinical condition. Third, because of the inconsistency of clinical record entries created by different clinicians across time, we could not correlate the volume changes we identified with consistently measured clinical outcomes. Fourth, decline in brain volume with time could confound our results, particularly in older individuals. However, reference to the published changes

\section{CONCLUSIONS}

Semiautomated volumetric analysis of the NCCTHs is reliable and detected a significant decrease in normalized brain volumes following mTBI in the setting of preinjury volumetric stability, suggesting neurodegeneration as the primary mechanism for volume loss. Further study is warranted to determine how these changes correlate with clinical outcomes.

\section{REFERENCES}

1. Faul M, Xu L, Wald MM, et al. Traumatic Brain Injury in the United States: Emergency Department Visits, Hospitalizations and Deaths 2002-2006. Atlanta: Centers for Disease Control and Prevention; 2010. https://www.cdc.gov/traumaticbraininjury/pdf/blue_book.pdf. Accessed July 19, 2017

2. Faul M, Coronado V. Epidemiology of traumatic brain injury. Handb Clin Neurol 2015;127:3-13 CrossRef Medline

3. Cassidy JD, Carroll LJ, Peloso PM, et al. Incidence, risk factors and prevention of mild traumatic brain injury: results of the WHO Collaborating Centre Task Force on Mild Traumatic Brain Injury. J Rehabil Med 2004;(43 Suppl):28-60 Medline

4. Centers for Disease Control and Prevention and National Center for Injury Prevention and Control. Report to Congress on Mild Traumatic Brain Injury in the United States: Steps to Prevent a Serious Public Health Problem. Atlanta: Centers for Disease Control and Prevention; 2003. https://www.cdc.gov/traumaticbraininjury/pdf/ mtbireport-a.pdf. Accessed July 19, 2017

5. Schmidt OI, Heyde CE, Ertel W, et al. Closed head injury: an inflammatory disease? Brain Res Brain Res Rev 2005;48:388-99 CrossRef Medline

6. Zhou Y, Kierans A, Kenul D, et al. Mild traumatic brain injury: longitudinal regional brain volume changes. Radiology 2013;267: 880-90 CrossRef Medline

7. Singh $\mathrm{P}$, Killgore WD. Time dependent differences in gray matter volume post mild traumatic brain injury. Neural Regen Res 2016;11: 920-21 CrossRef Medline

8. Wang X, Xie H, Cotton AS, et al. Early cortical thickness change after 
mild traumatic brain injury following motor vehicle collision. J Neurotrauma 2015;32:455-63 CrossRef Medline

9. Lui YW, Xue Y, Kenul D, et al. Classification algorithms using multiple MRI features in mild traumatic brain injury. Neurology 2014; 83:1235-40 CrossRef Medline

10. Zagorchev L, Meyer C, Stehle T, et al. Differences in regional brain volumes two months and one year after mild traumatic brain injury. J Neurotrauma 2016;33:29-34 CrossRef Medline

11. Meier TB, Bellgowan PS, Bergamino M, et al. Thinner cortex in collegiate football players with, but not without, a self-reported history of concussion. J Neurotrauma 2016;33:330-38 CrossRef Medline

12. Jarrett M, Tam R, Hernández-Torres E, et al. A prospective pilot investigation of brain volume, white matter hyperintensities, and hemorrhagic lesions after mild traumatic brain injury. Front Neurol 2016;7:11 CrossRef Medline

13. Tate DF, York GE, Reid MW, et al. Preliminary findings of cortical thickness abnormalities in blast injured service members and their relationship to clinical findings. Brain Imaging Behav 2014;8:102-09 CrossRef Medline

14. Mayer AR, Hanlon FM, Ling JM. Gray matter abnormalities in pediatric mild traumatic brain injury. J Neurotrauma 2015;32:723-30 CrossRef Medline

15. Monti JM, Voss MW, Pence A, et al. History of mild traumatic brain injury is associated with deficits in relational memory, reduced hippocampal volume, and less neural activity later in life. Front Aging Neurosci 2013;5:41 CrossRef Medline

16. MacKenzie JD, Siddiqi F, Babb JS, et al. Brain atrophy in mild or moderate traumatic brain injury: a longitudinal quantitative analysis. AJNR Am J Neuroradiol 2002;23:1509-15 Medline

17. Barbosa RR, Jawa R, Watters JM, et al; Eastern Association for the Surgery of Trauma. Evaluation and management of mild traumatic brain injury: an Eastern Association for the Surgery of Trauma practice management guideline. J Trauma Acute Care Surg 2012;73: S307-14 CrossRef Medline

18. Tavender EJ, Bosch M, Green S, et al. Quality and consistency of guidelines for the management of mild traumatic brain injury in the emergency department. Acad Emerg Med 2011;18:880 - 89 CrossRef Medline

19. McCrory P, Meeuwisse W, Dvořák J, et al. Consensus statement on concussion in sport-the 5 th international conference on concussion in sport held in Berlin, October 2016. Br J Sports Med 2017;51: 838-47 CrossRef Medline

20. Yushkevich PA, Piven J, Hazlett HC, et al. User-guided 3D active contour segmentation of anatomical structures: significantly improved efficiency and reliability. Neuroimage 2006;31:1116-28 CrossRef Medline

21. Shetty VS, Reis MN, Aulino JM, et al. ACR Appropriateness Criteria Head Trauma. J Am Coll Radiol 2016;13:668-79 CrossRef Medline

22. Allen JS, Damasio H, Grabowski TJ. Normal neuroanatomical variation in the human brain: an MRI-volumetric study. Am J Phys Anthropol 2002;118:341-58 CrossRef Medline
23. Fotenos AF, Mintun MA, Snyder AZ, et al. Brain volume decline in aging: evidence for a relation between socioeconomic status, preclinical Alzheimer disease, and reserve. Arch Neurol 2008;65:113-20 CrossRef Medline

24. Fjell AM, Walhovd KB, Fennema-Notestine C, et al. One-year brain atrophy evident in healthy aging. $J$ Neurosci 2009;29:15223-31 CrossRef Medline

25. Mac Donald CL, Barber J, Andre J, et al. 5-Year imaging sequelae of concussive blast injury and relation to early clinical outcome. $\mathrm{Neu}$ roimage Clin 2017;14:371-78 CrossRef Medline

26. Farbota KD, Sodhi A, Bendlin BB, et al. Longitudinal volumetric changes following traumatic brain injury: a tensor-based morphometry study. J Int Neuropsychol Soc 2012;18:1006-18 CrossRef Medline

27. Epstein DJ, Legarreta M, Bueler E, et al. Orbitofrontal cortical thinning and aggression in mild traumatic brain injury patients. Brain Behav 2016;6:e00581 CrossRef Medline

28. Bernick C, Banks S. What boxing tells us about repetitive head trauma and the brain. Alzheimers Res Ther 2013;5:23 CrossRef Medline

29. Reuter M, Schmansky NJ, Rosas HD, et al. Within-subject template estimation for unbiased longitudinal image analysis. Neuroimage 2012;61:1402-18 CrossRef Medline

30. Lewén A, Fredriksson A, Li GL, et al. Behavioural and morphological outcome of mild cortical contusion trauma of the rat brain: influence of NMDA-receptor blockade. Acta Neurochir (Wien) 1999; 141:193-202 CrossRef Medline

31. Farkas O, Povlishock JT. Cellular and subcellular change evoked by diffuse traumatic brain injury: a complex web of change extending far beyond focal damage. Prog Brain Res 2007;161:43-59 CrossRef Medline

32. Duning T, Kloska S, Steinsträter O, et al. Dehydration confounds the assessment of brain atrophy. Neurology 2005;64:548-50 CrossRef Medline

33. Scahill RI, Frost C, Jenkins R, et al. A longitudinal study of brain volume changes in normal aging using serial registered magnetic resonance imaging. Arch Neurol 2003;60:989-94 CrossRef Medline

34. Bernard C, Helmer C, Dilharreguy B, et al. Time course of brain volume changes in the preclinical phase of Alzheimer's disease. Alzheimers Dement 2014;10:143-51.el CrossRef Medline

35. Mathalon DH, Sullivan EV, Lim KO, et al. Progressive brain volume changes and the clinical course of schizophrenia in men: a longitudinal magnetic resonance imaging study. Arch Gen Psychiatry 2001; 58:148-57 CrossRef Medline

36. Guevara C, Bulatova K, Soruco W, et al. Retrospective diagnosis of parkinsonian syndromes using whole-brain atrophy rates. Front Aging Neurosci 2017;9:99 CrossRef Medline 